\title{
A Study On Quantitative Parameters OF SPECTRUM HANDOFF IN COGNITIVE RADIO NETWORKS
}

\author{
J. Josephine Dhivya ${ }^{1}$ and Ramaswami Murugesh ${ }^{2}$ \\ ${ }^{1}$ Research Scholar, Department of Computer Applications, Madurai Kamaraj University, \\ Madurai, India \\ ${ }^{2}$ Associate Professor, Department of Computer Applications, Madurai Kamaraj \\ University, Madurai, India
}

\begin{abstract}
The innovation of wireless technologies requires dynamic allocation of spectrum band in an efficient manner. This has been achieved by Cognitive Radio (CR) networks which allow unlicensed users to make use of free licensed spectrum, when the licensed users are kept away from that spectrum. The cognitive radio makes decision, switching from primary user to secondary user and vice-versa, based on its built-in interference engine. It allows secondary users to makes use of a channel based on its availability i.e. on the absence of the primary user and they should vacate the channel once the primary user re-enters and continue their communication on another available channel and this process in the cognitive radio is known as spectrum mobility. The main objective of spectrum mobility is that, there is no interruption caused due to the channel occupied by secondary users and maintains a good quality of service. In order to achieve better spectrum mobility, it is mandatory to choose an effective spectrum handoff strategy with the capability of predicting spectrum mobility. The handoff strategy with its parameters and its impact is an important concept in spectrum mobility but fairly explored. In this paper an empirical study on quantitative parameters involved in spectrum mobility prediction are discussed in detail. These parameters are studied extensively because they play a vital role in the spectrum handoff process moreover the impact of these parameters in various handoff methods can be used to predict the effectiveness of the system.
\end{abstract}

\section{KEYWORDS}

Cognitive Radio Networks, Spectrum Mobility and Spectrum Handoff.

\section{INTRODUCTION}

The increase in demand of data rate due to the transition of voice only communications to multimedia based applications requires opportunistic usage of spectrum which is not possible in the currently employed frequency allocation scheme [1]. An emerging solution to this problem is the Cognitive Radio (CR).It is an intelligent device with the capability of sensing environmental conditions and changes its parameters for optimized performance at network level [2].CR network is one that can identify current network condition and then decide and take action accordingly. CR network consists of three core tasks: radio scene analysis; channel identification; and transmit power control and dynamic spectrum management. A CR network is made up of primary and secondary users, coexisting together and contest to make use of the allotted 
spectrum. The problem of spectrum scarcity in fixed spectrum utilization is resolved by dynamic spectrum access via CR networks which provides the capability to use or share spectrum in opportunistic manner [3]. A dynamic spectrum sharing method needs to be devised to ensure fair spectrum sharing amongst both the primary and secondary users. Opportunistic users may dynamically select best available channels, and adapt their transmission parameters to avoid harmful interference between contending users. The $\mathrm{CR}$ has the ability to analyze the radio environment and to dynamically adapt its operating parameters to make the best use of the available spectrum. This is being achieved by equipping radio devices with enabling capabilities such as spectrum sensing, adaptive transmission, and software configurability. The CR networks ensure better usage of available spectrum to achieve higher end-to-end Quality of Service (QoS) in terms of throughput and/or delay performance. However, developing an algorithm and scheme for effective functioning CR network can be a challenging task. Medium Access Control has an important role in several CR functions that covers spectrum mobility, channel sensing, resource allocation, and spectrum sharing [5]. Spectrum mobility allows a secondary user to vacate its channel, when a primary user is enter into his allotted spectrum, and access to available band where it can re-establish the communication link [4]. Channel sensing is the ability of a cognitive user to collect information about spectrum usage, and to maintain a dynamic picture of available channels. Resource allocation is employed to opportunistically assign available channels to cognitive users according to QoS requests [6]. Spectrum access deals with contentions between heterogeneous primary and secondary users in order to avoid harmful interference.

\section{SPECTRUM MOBILITY}

In contrast to the various multi-channel wireless network technologies, the spectrum availability in CR networks varies dynamically with respect to time and space matters. In recent times, wireless networks incorporating spectrum technology, there is a vast demand for cognitive radio oriented network that reduce the problem of spectrum mobility that arises due to the concept of fixed spectrum allocation. Spectrum mobility is defined as the migration of the cognitive node over the spectrum and it is invoked when the secondary user moves in the transmission range of the primary user. In general the spectrum mobility in a mobile CR network is triggered by the behaviours of cognitive node mobility [13]. The most important and challenging problems in spectrum mobility are the coexistence of secondary users along with primary ones. Secondary user communication is often disrupted in the highly dynamic environments. Hence there is need to introduce spectrum mobility in the CR networks to enable seamless secondary user data transmission. For effective spectrum mobility the secondary user should find in advance the next available unused channel for continuing its transmission. In case of non-availability of free channels then path repair or rerouting will occurs. Hence CR has to continuously observe all the dynamic changes in the spectrum besides it incurs high energy cost. Therefore energy efficiency is also a primary factor taken into consideration for performance evaluation of CR networks.

\section{SPECTRUM HANDOFF}

In CR networks, the spectrum handoff can be defined as the process whereby a secondary user changes its operating frequency. The main problem in a change of frequency is the time it takes to find a new channel available and the type of information that is being transmitted. There are two users associated events takes place to initiate spectrum handoff in CR networks. First, the appearance of primary user in the licensed channel essentially makes secondary user to establish handoff. Second, spectrum handoff can happen because of secondary user mobility. In the second case, when CR user moves spatially, it may happen when transmission coverage of the cognitive 
International Journal of Wireless \& Mobile Networks (IJWMN) Vol. 9, No. 1, February 2017

user overlaps with a licensed user currently using same channel [9]. Spectrum handoff can be explained as a cyclic process and it has two phases: Evaluation phase and Link maintenance phase. In the evaluation phase, cognitive user observes the situation and analyses whether handoff triggering incident shall take place or not. Once secondary user chooses to perform spectrum handoff, it enters link maintenance phase. In this phase, cognitive user hands over the channel to the licensed user and maintains data transmission over another available channel. Finally secondary vacates the link maintenance phase and then continues cycle.

\section{TYPE OF SPECTRUM HANDOFF}

The spectrum handoff ( $\mathrm{SHO}$ ) can be defined as the process whereby a secondary user changes its operating frequency. Handoff strategies are broadly classified into four categories: handoff triggering-timing based, mobility based spectrum aware, probability based and spectrum sensing based.

\subsection{Handoff triggering timing based SHO}

The handoff triggering timing based SHO functionality is based on the timing when the spectrum sensing and spectrum handoff triggering event occurs and hence this type of handoff scheme represents the timing instant for SHO. Hattab et al, Jang and Umar et al [7, 8, 9] studied extensively the performance of timing based SHO. This type of SHO is further classified into reactive, proactive and hybrid spectrum handoff [10]. In reactive method the free channel is sensed first then the handoff action is performed and finally the channel switching is done after identifying the free channel.

The advantage of reactive spectrum handoff scheme is that CR can obtain the accurate target channel. Hence, for short sensing time, reactive spectrum handoff performs better than proactive spectrum handoff scheme. If the sensing time is large, then it will result in larger handoff latency. Hence, reactive spectrum handoff scheme perform worse than proactive spectrum handoff scheme in terms of extended data delivery time when the sensing time is large [11].

In case of proactive method the sensing for free channel and the handoff action are performed in proactive manner in CR networks. The target free channel for future spectrum handoff is based on primary user traffic. Hence the handoff latency is reduced to a great extent compared to reactive spectrum handoff scheme. An efficient proactive spectrum handover mechanism using packet scheduling algorithms to reduce unusable channel was proposed which not only reduces the bandwidth fragmentation and improves the channel utilization but also minimizes the packet loss probability and total service time for CRN [12].A proactive spectrum access scheme using channel histories was proposed [13] to make future spectrum availability and schedule the channel usage in advance. The hybrid method is in general the combination of reactive and proactive spectrum handoff method [11].

\subsection{Mobility Based SHO}

Mobility based SHO focuses on dynamic spectrum management, user mobility management and resource allocation for CR networks which is best suited for CR cellular networks. The user mobility management is used to select the proper handoff scheme to minimize the switching latency at the cell boundary. A novel CR network architecture based on the spectrum pooling 
concept is proposed which is a suitable structure for operating in dynamic radio environment for handling both spectrum and user mobility for CR cellular networks [14].

\subsection{Probability Based SHO}

The probability based handoff method is used for identifying channel conditions thereby enabling the CR network to find whether the channel available or not. A probability based method is applied to determine the initial and target channel to carry out the decision process [15]. The main features in this type of handoff are it minimizes extended service time and average break time for the secondary connection, reduces collision probability between CRs and suitable for well modeled primary users low handoff latency. Three probability thresholds to be considered: threshold below - which current channel is considered busy at the end of frame transmission; threshold above - which channel is idle at the end of the current frame; and threshold above which channel is idle for the next frame transmission

\subsection{Spectrum Sensing Based SHO}

This type of handoff is based on first selecting the appropriate channel for each secondary user and then determining the sensing order with the target handoff channel by means of dynamic programming. The key feature in this type of SHO is the number of spectrum handoff is reduced significantly thereby increasing the performance of a system [16].

\section{SPECTRUM HANDOFF PERFORMANCE INDICATORS}

The performances of spectrum handoff algorithms are not homogeneous and it order to select ideal handoff algorithm for particular environment, we need to evaluate its performances with certain performance indicators. The spectrum handoff performance indicators determine the overall efficiency of CR networks. The following indicators help us to improve the performance of SHO with minimal collision.

\subsection{Number of Spectrum Handoff (NSH)}

NSH is defined as the number of handoffs that occurs in CR networks during one session of CR data transmission.CR networks are considered to be efficient if and only if the number of handoff occurring are minimal [10].This indicator is investigated in the interference-limited resource optimization in cognitive femto cells with fairness and imperfect spectrum sensing[17]. It is also proposed and investigated that this value can be effectively reduced by a delay requirement [19] and spectrum usage [18].

\subsection{Cumulative handoff delay (CHD)}

This parameter in general refers to the delay occurrence in spectrum handoff in CR [20].The handoff latency can be estimated from various parameters such as handoff preparation time, time to determine the proper spectrum band out of available spectrum bands, sensing synchronization time and spectrum switching delay and inter cell resource allocation delay for CR cellular networks [21]. 
International Journal of Wireless \& Mobile Networks (IJWMN) Vol. 9, No. 1, February 2017

\subsection{Link maintenance probability (LMP)}

The LMP is defined as the probability that the link is successfully maintained in a hop or between troubled nodes, which is dependent on the probability of channel availability between two nodes. Link maintenance probability indicates that the probability that the communication link is successfully maintained for successful data transmission within the allowable spectrum handoff in CR networks [10].This probability parameter was investigated for effective SHO in case of adhoc networks in CR for seamless spectrum sensing and effective channel usage [19]. Moreover, LMP parameter was observed and investigated under spectrum sharing using femto cells to enable packet transfer without loss [21]. The spectrum handoff process takes place when the current channel does not successfully send the data. Also spectrum handoff does not occur when the transmission on any of all of the available channels fails.

\subsection{Effective Data Rate (EDR)}

EDR indicator is defined as the average amount of data which is successfully transferred between two nodes which are available in CR network and also maintains link maintenance probability. In [22], EDA was investigated and result shows that the frequency of handover can be reduced and secondary user utilization can be increased subsequently. The maximal data rate for any secondary user operating in spectrum band is represented by

$$
D_{r}=W_{c 1} \log _{2}\left(1+\frac{P_{T} G_{c 1}}{n_{0}}\right)
$$

$\mathrm{W}_{\mathrm{c} 1}$ denotes the bandwidth of the channel of type $1, \mathrm{n}_{0}$ is the power of the additive white Gaussian noise, $\mathrm{P}_{\mathrm{T}}$ is the transmission power for secondary user and $\mathrm{G}_{\mathrm{c} 1}$ is the channel gain of type 1 .

\subsection{Probability of misdetection and false alarm (PMFA)}

PMFA is one of the primary indicators that have to be avoided for efficient handoff without collision in CR networks and occurrence of this parameter PMFA causes interference and improper usage of spectrum in CR networks [23]. Efficient sensing orders proposed in [24, 25] are highly sensitive to false alarm and misdetection probabilities. The misdetection probability $\left(P_{m}\right)$ and false alarm probability $\left(P_{f}\right)$ in CR networks [33] are defined as

$$
\begin{aligned}
& P_{m}=P\left(H_{0} \mid H_{1}\right) \\
& P_{f}=P\left(H_{1} \mid H_{0}\right)
\end{aligned}
$$

where $\mathrm{H}_{0}$ - hypothesis representing channel is available for secondary user and $\mathrm{H}_{1}$ - hypothesis representing channel is currently occupied by primary user.

\subsection{Spectrum Handoff Ratio (SHR)}

The spectrum handoff ratio (SHR) is directly proportional to the spectrum handoff. For efficient spectrum handoff in CRN the value of this ratio should be minimal [22]. 
International Journal of Wireless \& Mobile Networks (IJWMN) Vol. 9, No. 1, February 2017

\subsection{Bandwidth Utilization (BWU)}

An efficient method of channel utilization can be referred to as bandwidth utilization. This is considered to play a vital role in performing spectrum handoff as it is responsible for channel allocation and is determining the overall performance of CRN [26].

\subsection{Energy Consumption (EC)}

Frequent occurrence of spectrum handoff results in inefficient usage of spectrum there by increasing the energy consumption. The concept of excessive channel switching should be optimized to reduce energy consumption in CR networks [27, 30].To enable secondary user to achieve maximum energy efficiency an optimal sensing order design has been proposed by applying a dynamic programming solution [28-29].

\subsection{Collision Probability (CP)}

There is an occurrence of collision in spectrum handoff whenever a primary user arrives on the channel used by a secondary user. The term collision probability is defined as the probability of having a collision between primary user and secondary user [30].For efficient spectrum usage and smooth handoff this value must be less.

\subsection{Blocking probability and forced termination probability (BPFTP)}

The blocking probability parameter represents the probability of not allowing the secondary user to use the network whenever the channel is unavailable or on hold. The forced termination probability parameter represents the probability of forcing the secondary user to vacate the channel for incoming primary user. In [32] an analysis of this parameter in performance analysis of cognitive radio spectrum access with prioritized traffic was made. In [33] investigation was made in modeling and analysis of spectrum handoffs for real-time traffic in cognitive radio networks. In [31] this parameter was investigated in Spectrum handoff scheme based on recommended channel sensing sequence. The forced termination probability [34] $\mathrm{P}_{\mathrm{f}}$ of a cognitive user can be defined as

$$
P_{f}=\frac{\sum_{\left(s, s^{\prime}\right) \in T} \pi_{s} \gamma_{s^{\prime}}^{\mathrm{s}}}{\left(1-P_{b}\right) \lambda_{c}}
$$

where $\mathrm{T}$ is the set that contains all state pairs $\left(s, s^{\prime}\right)$ in which a user is forced to terminate when transitioning from $\mathrm{s}$ to $\mathrm{s}$ 'and $P_{b}$ is the blocking probability. Formally, $\mathrm{T}$ can be defined as

$$
\mathrm{T}=\left\{\left(\mathrm{s}, \mathrm{s}^{\prime}\right)=\left(\left(\mathrm{i}_{1}, \mathrm{i}_{\mathrm{m}}\right),\left(\mathrm{i}_{1}^{\prime}, \mathrm{i}_{\mathrm{m}}^{\prime}\right)\right) \mid \mathrm{N}_{\mathrm{c}}(\mathrm{s})>\mathrm{N}_{\mathrm{c}}\left(\mathrm{s}^{\prime}\right) \text { and } \mathrm{N}_{\mathrm{p}}(\mathrm{s})<\mathrm{N}_{\mathrm{p}}\left(\mathrm{s}^{\prime}\right)\right\}
$$

where $\mathrm{N}_{\mathrm{c}}(\mathrm{s})$ and $\mathrm{N}_{\mathrm{c}}\left(\mathrm{s}^{\prime}\right)$ are the numbers of cognitive users in state $\mathrm{s}$ and $\mathrm{s}^{\prime}$ respectively, and $\mathrm{N}_{\mathrm{p}}(\mathrm{s})$ and $\mathrm{N}_{\mathrm{p}}\left(\mathrm{s}{ }^{\prime}\right)$ are the numbers of primary users in state $\mathrm{s}$ and $\mathrm{s}^{\prime}$ respectively[35]. The blocking probability $P_{b}$ is defined as

$$
P_{b}=\sum s \in B \frac{\pi_{s \lambda_{c}}}{\sum_{s \in s^{\prime}, s \neq s^{\prime}} \gamma_{s^{\prime}}^{s}}
$$


where $\mathrm{B}$ is the set of all the states in which blocking occurs when a new cognitive user arrives to the system, and is defined as $B=\left\{s=\left(i_{1} \ldots i_{m}\right) \mid \forall j 1 \leq j \leq m,-1<i_{j}<n\right\}$.

\section{CONCLUSION}

The valuable resource in wireless communication systems is spectrum which is widely focused area of research over the few decades. There are inevitable demands of extra frequency bands in wireless networking, CR networks provides an immense unused potential to wireless systems and spectrum handoff is very important but fairly explored area in CR networks. In this paper, a systematic overview on spectrum handoff methods and performance metrics are presented which thereby paves ways to utilize channels ensuring smooth and fast transmission. Future works include designing an intelligent handoff method including quantitative parameters that can increase the efficiency of the system.

\section{REFERENCES}

[1] Tevfik et al (2009), "A Survey of Spectrum Sensing Algorithms for Cognitive Radio Applications", ÏEEE Communications Surveys \& tutorials, Vol. 11, No. 1, first quarter.

[2] Ayubi Preet et al (2014), "Review paper on Cognitive Radio Networking and Communications", International Journal of Computer Science and Information Technologies, Vol. 5, No. 4,pp 55085511

[3] Harada, Alemseged Filin \& Riegel et al,(2013), "IEEE dynamic spectrum access networks standards committee”, IEEE Commun Magazine, Vol. 51, No. 3, pp104-111.

[4] Kumud Tiwari, \& Ashutosh Rastogi, (2016), "Network Spectrum Handoff in Cognitive Radio", International Journal of Advanced Research in Computer and Communication Engineering, Vol. 5, No. 4, pp 1025 - 1030.

[5] Lu Li, Yanming Shen, \& Keqiu Li, Kai Lin,(2011), “TPSH: A Novel Spectrum Handoff Approach Based on Time Estimation in Dynamic spectrum networks", Computational Science and Engineering (CSE), pp. $345-350$.

[6] Antonio De Domenico, Emilio Calvanese Strinati, \& Maria-Gabriella Di Benedetto,(2012), " A Survey on MAC Strategies for Cognitive Radio Networks" Vol. 14, No. 1, pp 21 - 44.

[7] Ghaith Hattab, \& Mohamed Ibnkahla, (2014), "Multiband Spectrum Access: Great Promises for Future Cognitive Radio Networks", Proceedings of the IEEE, Vol. 102, No. 3, pp 282 - 306.

[8] Won Mee Jang, (2014), "Blind Cyclostationary Spectrum Sensing in Cognitive Radios", IEEE communications, Vol. 18, No. 3, pp 393 - 396.

[9] Umar \& Sheikh AUH, (2013), "Unveiling the hidden assumptions of energy detector based spectrum sensing for cognitive radios", Physical Communication, Vol. 9, pp 148 - 170.

[10] Christian \& Chung, (2013), "Spectrum Mobility in Cognitive radio Networks", IEEE Communication Magazine, Vol.50, No.2, pp 114-121.

[11] Wang \& Wang, (2012), "Analysis of reactive spectrum handoff in Cognitive radio Networks", IEEE Communications, Vol.30, No.10, pp 28 - 30.

[12] Ma B \& Xie X, (2014), "An efficient proactive spectrum handover mechanism in cognitive radio networks", Wireless Personal Communications, Vol.79, No.3, pp 679-701.

[13] Yang \& Zheng, (2008), "Proactive channel access in dynamic spectrum networks" Physical Communications, Vol.1,pp $103-111$.

[14] Lee \& Akyildiz, (2012), "Spectrum-aware mobility management in cognitive radio cellular networks", IEEE Transactions on Mobile Computing, Vol.10, No.2, pp 161-174.

[15] Sheikholeslami \& Ashtiani,(2015), "Optimal probabilistic initial and target channel selection for spectrum handoff in cognitive radio networks", IEEE Transactions on Wireless Communications, Vol.14,No., 1 pp $570-584$.

[16] Wu \& Lingge, (2013), "Spectrum handoff scheme based on recommended channel sensing sequence" IEEE China Communications, Vol.10, No.8, pp 18 - 26. 
International Journal of Wireless \& Mobile Networks (IJWMN) Vol. 9, No. 1, February 2017

[17] Zhang \& Chen, (2016), "Interference-limited resource optimization in cognitive femto cells with fairness and imperfect spectrum sensing”, IEEE Transactions on Vehicular Technology,Vol.65,No.3, pp $1761-1771$.

[18] Lertsinsrubtavee \& Malouch,(2012), "Controlling spectrum handoff with a delay requirement in cognitive radio networks", Proceedings of the international conference on computer communication and networks, pp 1-8.

[19] Kalil \& Mitschele (2010), "A Spectrum handoff reduction for cognitive radio ad hoc networks", Proceedings of the 7th international symposium on wireless communication systems, pp $1036-1040$.

[20] Zhang \& Song, (2012), "Modeling for spectrum handoff based on secondary users with different priorities in cognitive radio networks", Proceedings of the international conference on Wireless Communications and signal processing, pp 1-6.

[21] Zhang \& Tao, (2014), "Resource allocation in spectrum sharing OFDMA femto cells with heterogeneous services", Proceedings of the IEEE, Vol. 62, No. 7, pp $2366-2377$.

[22] Lu \& Fan, (2010), "Adaptive power control based spectrum handover for cognitive radio networks", Proceedings of the IEEE Wireless communication and Networking Conference, pp $1-5$.

[23] Qiao \& Tan, (2011), "Combined optimization of spectrum handoff and spectrum sensing for cognitive radio systems", Proceedings of the IEEE Wireless communication, networking and mobile computing, pp $1-4$.

[24] Song \& Xie, (2012), "Prospect: A proactive spectrum handoff framework for cognitive radio ad hoc networks without common control channel," IEEE Transactions on Mobile Computing, Vol. 11,No. 7, pp. 1127-1139.

[25] Khan \& Latvaaho, (2013), “Autonomous sensing order selection strategies exploiting channel access information,” IEEE Transactions on Mobile Computing, Vol. 12, No. 2, pp. 274-288.

[26] Xian \& Xu, (2012), "A grade-based spectrum handover mechanism in cognitive radio system", Recent advances in Computer Science and Information Engineering Springer link,Vol.127, pp 327 332 .

[27] Wang \& Coon, (2013), "Reliable energy-efficient spectrum management and optimization in cognitive radio networks: how often should we switch", IEEE Wireless communication, Vol.20, No.6, pp 14- 20.

[28] Pei \& Liang, (2011), "Energy-efficient design of sequential channel sensing in cognitive radio networks: optimal sensing strategy, power allocation, and sensing order" IEEE Journal on selected areas in communication, Vol.28, No.8, pp $1648-1659$.

[29] Eryigit \& Bayhan, (2013), "Energy- efficient multichannel cooperative sensing scheduling with heterogeneous channel conditions for cognitive radio networks" ,IEEE Transactions on Vehicular technology,Vol.62,No.6, pp 2690 - 2699 .

[30] Urkowitz (2000), "Energy detection of unknown deterministic signals," Proceedings of IEEE, Vol. 55 , pp. 523-531.

[31] Song \& Xie, (2011), "Performance analysis of spectrum handoff for cognitive radio ad hoc networks without common control channel under homogeneous primary traffic", Proceedings of IEEE INFOCOM, pp. 3011 - 3019.

[32] Tumuluru \& Niyato,(2012), "Performance analysis of cognitive radio spectrum access with prioritized traffic", IEEE Transactions on Vehicular Technology, Vol.61, No.4, pp 1895 -1906.

[33] Hou \& Yeung, (2013), "Modeling and analysis of spectrum handoffs for real- time traffic in cognitive radio networks" Proceedings of IEEE published in the First International Symposium on Computing and Networking, pp 415-421 .

[34] Letaief \& Zhang, (2009), "Cooperative communications for cognitive radio networks" Proceedings of IEEE, Vol. 97, No.5, pp 878-893.

[35] B Modi \& A Annamalai, "Ergodic capacity analysis of cooperative amplify-and-forward relay networks over Rice and Nakagami fading channels". International Journal of Wireless \& Mobile Networks, Vol. 4 (1), pp 99-116. 